\title{
Residual protective effect of some intermediates in polyvinylpyrrolidone production
}

\author{
N. A Belousova,* V. P. Grigor'ev and E. V. Plekhanova \\ Southern Federal University, Zorge st. 7, Rostov-on-Don, 344090 Russian Federation \\ *E-mail:nina.a.belousova@gmail.com
}

\begin{abstract}
The regularities of changes in the residual protective effect (RPE) of wastes of polyvinylpyrrolidone production on the acid corrosion of steel were investigated. The duration of RPE increases with increasing duration of pre-adsorption. It was shown that the duration of the $\operatorname{RPE}(\tau)$ is a function of the additive concentration in the pre-adsorption solution.
\end{abstract}

Key words: inhibitor, corrosion of steel, residual protective effect, near-electrode concentration, effective activation energy.

Received: December 10, 2014.

doi: $\underline{10.17675 / 2305-6894-2015-4-1-049-056}$

\section{Introduction}

A long-lasting residual protective effect (RPE) of the inhibitor is important for some technologies. The use of additives with a prolonged RPE allows reducing the discharge of the inhibitor during transportation of corrosive media through pipes with periodic recharge of these media with the respective inhibitor. In addition, it allows protecting metal items during interoperation transportation in production. Previously it has been shown that semiproducts of industrial production of polyvinylpyrrolidone are effective inhibitors of corrosion of steel, nickel and aluminum in $1 \mathrm{M}$ hydrochloric acid [1,2]. Therefore it is of interest to study the parameters of the RPE for wastes of PVP production.

\section{Objects of the study and experimental procedure}

We determined the RPE of inhibitor additives from the mass loss of specimens (at least three specimens per point). Specimens of low carbon steel St3 with dimensions of $10.0 \times 12.5 \mathrm{~mm}$ cut from steel sheet were degreased with ethanol, then chemically degreased by boiling for 5 minutes in a solution with the following composition, g/l: 10 $\mathrm{NaOH}, 25 \mathrm{Na}_{3} \mathrm{PO}_{4}, 3 \mathrm{Na}_{2} \mathrm{SiO}_{3}$. Prepared steel electrodes were weighted and transferred to the respective pre-adsorption solution. After exposure for 12 or $36 \mathrm{~h}$ the electrodes were washed in distilled water, dried with filter paper and immersed in $1 \mathrm{M} \mathrm{HCl}$ solutions at temperatures of $20-50^{\circ} \mathrm{C}$. After each subsequent period $t$, the next three electrodes were retrieved, washed, dried with filter paper and weighted to estimate the RPE parameter. 
Two characteristics were estimated:

$K_{t}$ - the RPE coefficient after time $t$ from the start of corrosion in $1 \mathrm{M} \mathrm{HCl}$ solution (without an inhibitor) - the reference solution:

$$
K_{t}=\frac{\Delta m_{0}}{\Delta m_{i}},
$$

where $\Delta m_{0}$ is the mass loss of the specimen over time $t$ from the instant of its immersion in the $\mathrm{HCl}$ solution; $\Delta m_{i}$ is the same value for an electrode pretreated with an inhibitor.

$\mathrm{Z}_{t}(\%)$ - the degree of protection:

$$
Z_{t}=\left(1-\frac{1}{K_{t}}\right) \cdot 100 \%
$$

\section{Measurement results and discussion}

As shown in [3,4], the dependence of RPE time $v s$. the logarithm of inhibitor additive concentration for individual substances is linear. Wastes of PVP production are complex mixtures of various substances, and competitive adsorption of the components of these mixtures during the pretreatment is possible, so the $\tau-\lg C$ function may differ from linearity. In case of the PVP-1 additive, this dependence is linear (Figure 1), but the duration of the RPE is the same at PVP-1 concentration of 5 and $10 \mathrm{~g} / \mathrm{l}$ in the preadsorption solution. Apparently, a limit of the additive adsorption is reached at the concentration of $5 \mathrm{~g} / \mathrm{l}$ and further addition of PVP-1 does not result in additional build-up and densification of the adsorption layer on the metal surface.

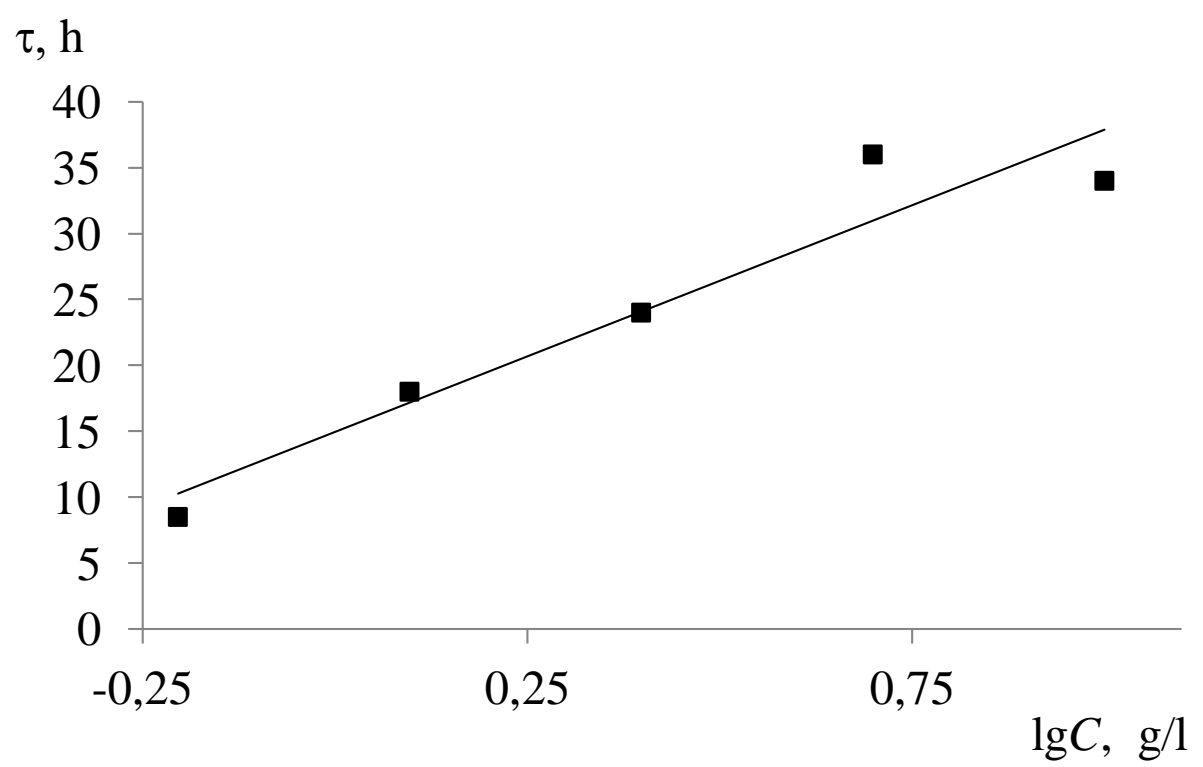

Figure 1. Dependence of the residual protective effect duration $\tau$ of PVP-1 on concentration at $20^{\circ} \mathrm{C}$. 
The PVP-1 additive behaves like a typical adsorption inhibitor and the degree of the metal protection decreases with decreasing concentration of the additive in solution. A similar dependence was observed for the RPE time, which decreased with decreasing concentration of the additive (Figure 1). Thus after a 12 hour exposure of metal specimens in the pre-adsorption solution with PVP-1 concentrations of 5 and $10 \mathrm{~g} / \mathrm{l}$, the inhibitor was completely desorbed from the metal surface after 34 hours. The RPE duration decreased to 8.5 hours when the additive concentration was $0.625 \mathrm{~g} / \mathrm{l}$.

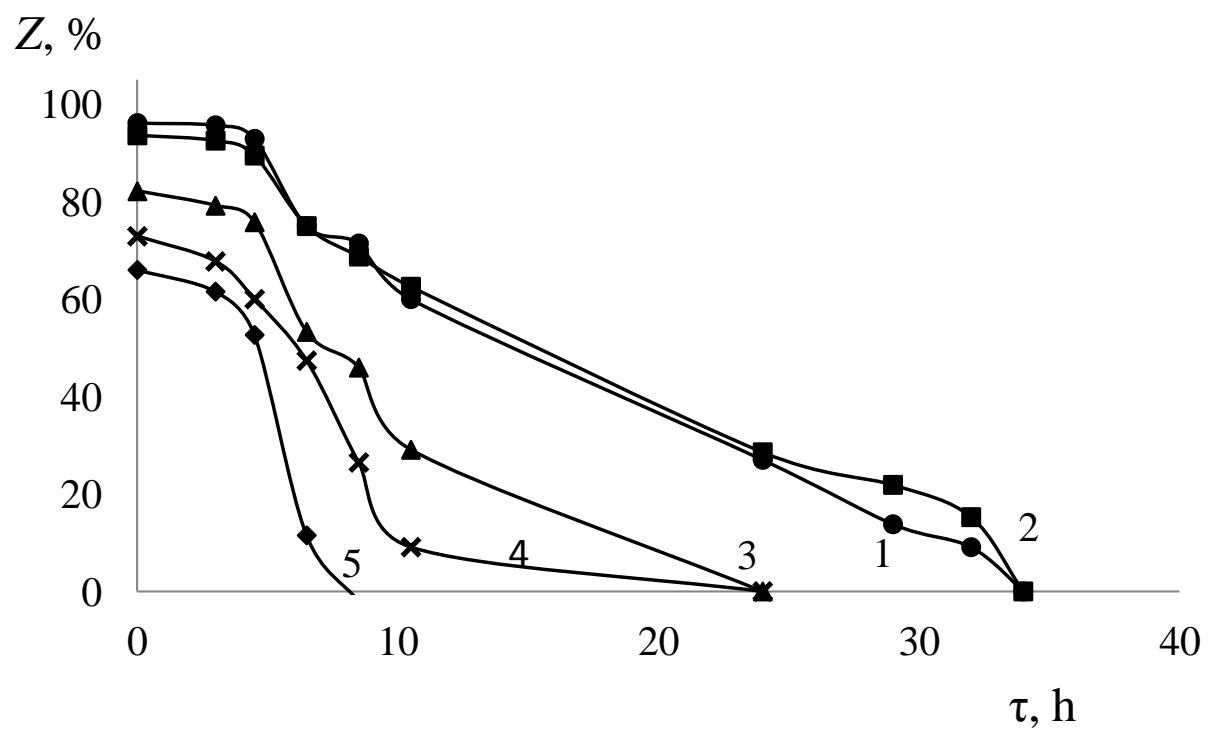

Figure 2. Reducing the degree of protection over time at various concentrations of PVP-1 in the pre-adsorption solution : $1-10 \mathrm{~g} / 1,2-5 \mathrm{~g} / 1,3-2.5 \mathrm{~g} / \mathrm{l}, 4-1.25 \mathrm{~g} / \mathrm{l}, 5-0.625 \mathrm{~g} / \mathrm{l}$.

For all the concentrations studied, two sections can be distinguished in the $Z_{\tau}-t$ curve. The first section of a curve is located between 0 and about 5 hours. It corresponds to destruction of a layer of particles formed due to weak Van der Waals interaction forces. Desorption of physically adsorbed particles occurs in the second section. The length of this section decreases with decreasing concentration of the additive.

Duration of RPE increases from 34 to 130 hours at a PVP-1 concentration of $10 \mathrm{~g} / \mathrm{l}$ by prolonging the pre-adsorption to 36 hours.

Obviously, the change in the protective action of the inhibitor under RPE conditions is associated with continuous desorption of the surfactant from the electrode surface. The changes in time of the inhibitor near-electrode concentration in the reference solution lead to the corresponding changes in $K_{\tau}$, steady-state corrosion potential, and effective activation energy. Thus, it is necessary to estimate the near-electrode concentration of inhibitor $C_{\mathrm{s}}$ as a function of time, i.e., $C_{\mathrm{s}}=f(t)$. The subsequent discussion of experimental data is based on the assumption that the plot of corrosion inhibition coefficient $K$ on the inhibitor concentration is the same under steady-state corrosion conditions and in the case of desorption, i.e. $K_{\tau}=f(C)$. In this case, based on the experimental dependence $K=f(C)$ for steady-state conditions and known $K_{\tau}$ values for various $t$ values, it is possible to 
determine the corresponding concentrations $C_{\mathrm{s}}$ under RPE conditions and hence to find various electrochemical characteristics under desorption conditions as functions of concentration $C_{\mathrm{s}}$ for various points of time $t$ from the start of desorption of the surfactant. Figure 3 shows the logarithmic dependence of the corrosion inhibition coefficient on the concentration of the additive. The linearity of the relationship allows us to obtain the dependence shown in Figure 4.

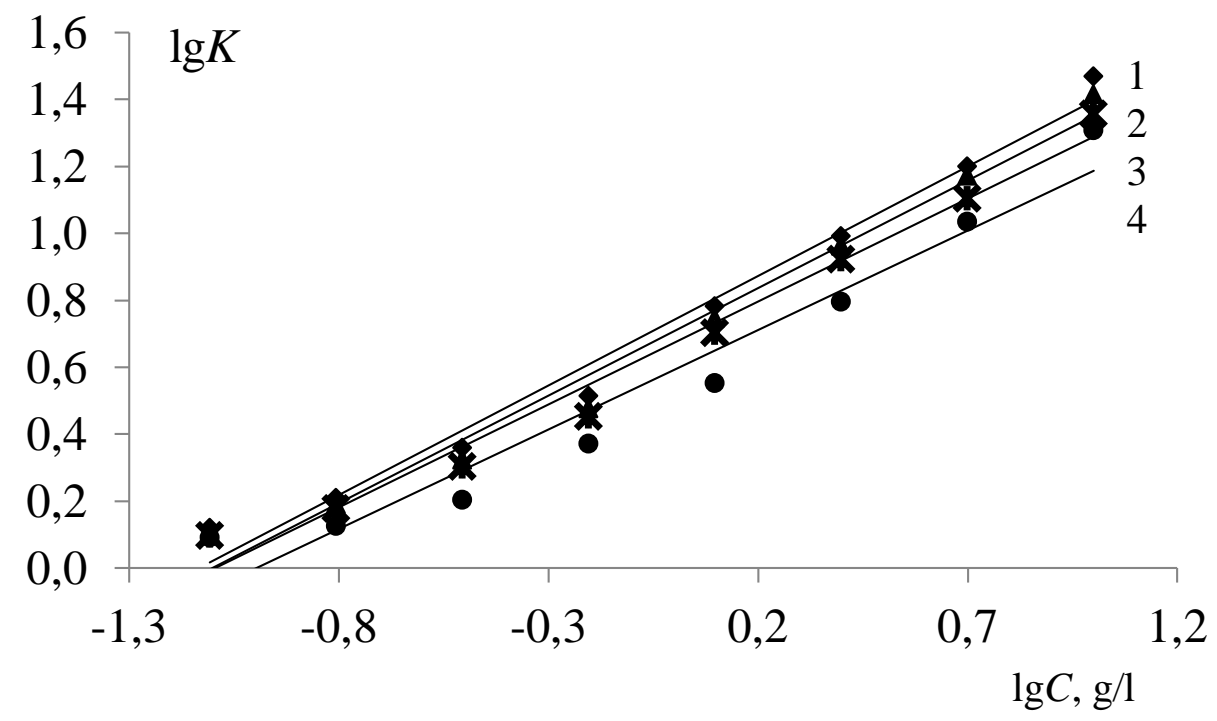

Figure 3. Logarithmic dependence of the corrosion inhibition coefficient for steel in $1 \mathrm{M} \mathrm{HCl}$ on the concentration of PVP-1 at temperatures: $1-20^{\circ} \mathrm{C}, 2-30^{\circ} \mathrm{C}, 3-40^{\circ} \mathrm{C}, 4-50^{\circ} \mathrm{C}$.

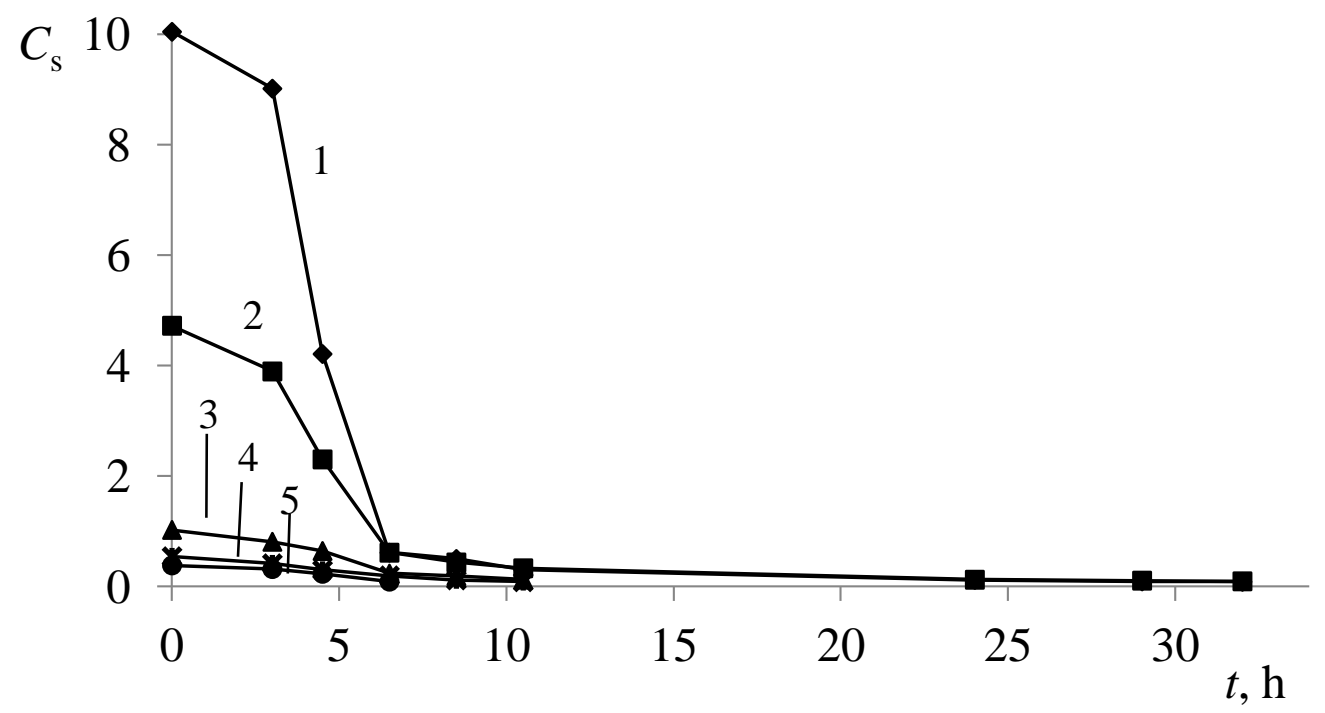

Figure 4. Dependence of the near-electrode concentration of PVP-1 under RPE conditions for steel versus time in $1 \mathrm{M} \mathrm{HCl}$ after treatment in pre-adsorption solution at concentrations: 1 $10 \mathrm{~g} / \mathrm{l}, 2-5 \mathrm{~g} / \mathrm{l}, 3-2.5 \mathrm{~g} / \mathrm{l}, 4-1.25 \mathrm{~g} / \mathrm{l}, 5-0.625 \mathrm{~g} / \mathrm{l}$.

As expected, the near-electrode concentration of the additive depends on the concentration of such in pre-adsorption solution. The lower the concentration of PVP-1 in 
the pre-adsorption solution, the smaller the time of complete desorption of the inhibitor from the metal surface becomes.

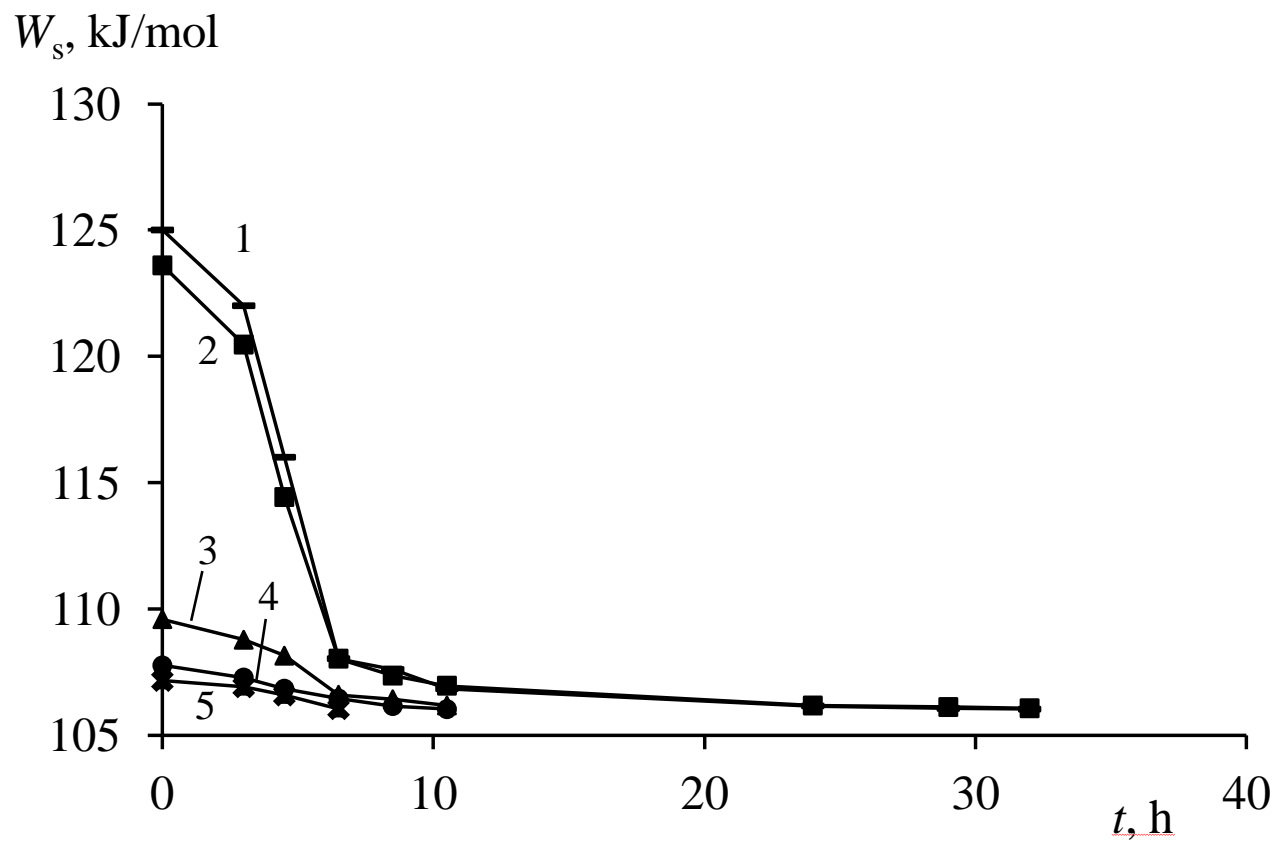

Figure 5. Dependence of the effective activation energy of PVP-1 under RPE conditions for steel versus time in $1 \mathrm{M} \mathrm{HCl}$ after treatment in pre-adsorption solution at concentration: 1 $10 \mathrm{~g} / \mathrm{l}, 2-5 \mathrm{~g} / \mathrm{l}, 3-2.5 \mathrm{~g} / \mathrm{l}, 4-1.25 \mathrm{~g} / \mathrm{l}, 5-0.625$.

Figure 5 shows the dependence of the effective activation energy on the nearelectrode concentration in RPE. The figure shows that the activation energy is reduced at first after immersion in the reference solution. Later, however, the value of $W$ is almost constant and is in the range from 106 to $125 \mathrm{~kJ} / \mathrm{mol}$.

For PVP-2 and PVP-3 inhibitors, whose protective effect has a thermal peak, it is of interest to study the effect of temperature of the reference solution on the variation of the degree of protection in time.

The maximum protective effect of PVP-2 is observed by $50^{\circ} \mathrm{C}$ under RPE conditions. The maximum degree of protection is achieved at $30^{\circ} \mathrm{C}$. The experiment was terminated before complete desorption of the inhibitor from the metal surface, because active butt corrosion on the steel specimens immersed in the reference solution was observed in $130 \mathrm{~h}$ at $20^{\circ} \mathrm{C}$, in $48 \mathrm{~h}$ at $30^{\circ} \mathrm{C}$, in $28 \mathrm{~h}$ at $40^{\circ} \mathrm{C}$, and in $8 \mathrm{~h}$ at $50^{\circ} \mathrm{C}$. On the curve $Z_{\mathrm{t}}-t$ one can distinguish sections corresponding to desorption of physically adsorbed and chemisorbed particles. The desorption of physically adsorbed particles occurs before 32 hours at $20^{\circ} \mathrm{C}$ or 36 hours at $30^{\circ} \mathrm{C}$. It is not possible to distinguish the sections corresponding to desorption of physically adsorbed and chemisorbed particles at temperatures of $40^{\circ} \mathrm{C}$ and $50^{\circ} \mathrm{C}$ because the experiment was finished before complete desorption of PVP-2. 


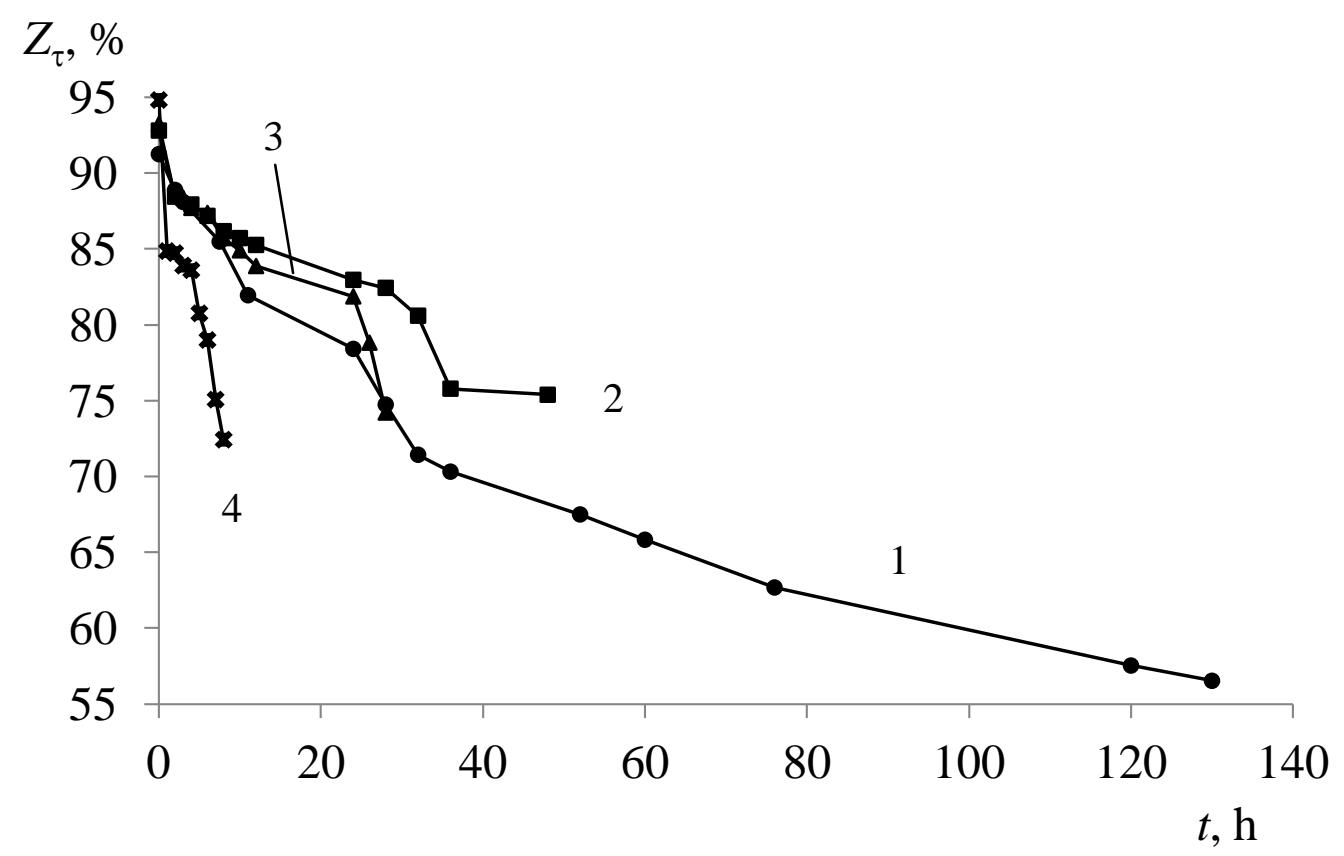

Figure 6. The decrease in the degree of protection over time under RPE conditions for steel in $1 \mathrm{M} \mathrm{HCl}$ after pretreatment with PVP-2 at temperatures of the reference solution: $1-20^{\circ} \mathrm{C}$, $2-30^{\circ} \mathrm{C}, 3-40^{\circ} \mathrm{C}, 4-50^{\circ} \mathrm{C}$.

Addition of PVP-3 has a maximum protective effect at $50^{\circ} \mathrm{C}$, but the duration of the RPE decreases upon increasing the temperature of the desorption solution (Figure 7).

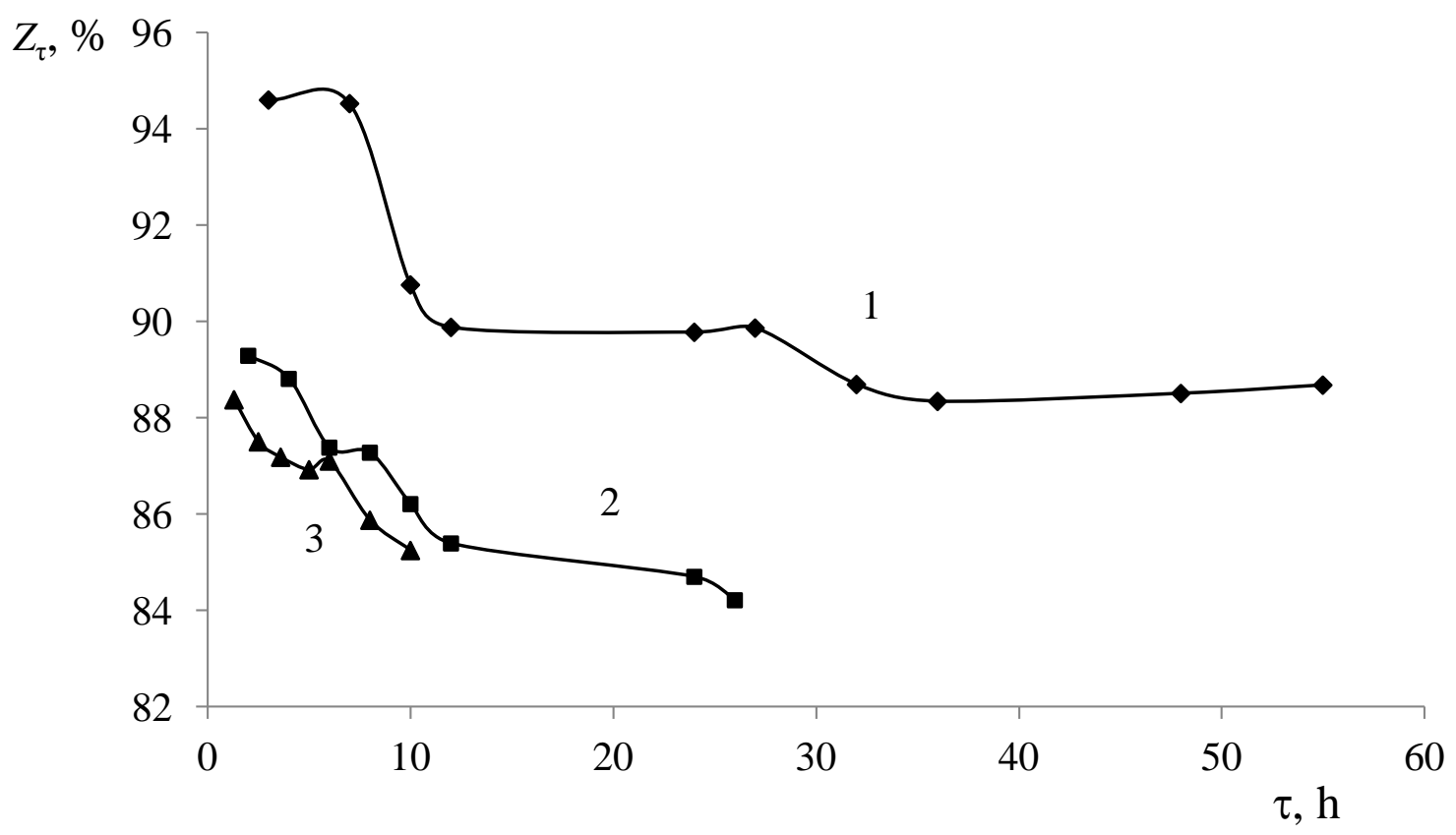

Figure 7. The degree of protection versus time for the additive PVP-3 at temperatures: 1 $30^{\circ} \mathrm{C}, 2-40^{\circ} \mathrm{C}, 3-50^{\circ} \mathrm{C}$. 
The graphical time dependence of the degree of protection $Z_{\tau}$ (Figure 7) calculated on the basis of $K_{\tau}$ values under RPE conditions demonstrates a difference in the shapes of the curves at different temperatures. In this case the experiment was also terminated before reaching a degree of protection $0 \%$ or at least $50 \%$ as active butt corrosion on the steel specimens immersed in the reference solution was observed in $55 \mathrm{~h}$ at $30^{\circ} \mathrm{C}$, in $27 \mathrm{~h}$ at $40^{\circ} \mathrm{C}$, and in $10 \mathrm{~h}$ at $50^{\circ} \mathrm{C}$. The minimal degree of protection in these cases was $88.7 \%$, $84.2 \%$ and $85.2 \%$, accordingly. Visible butt corrosion on pre-treated specimens was observed only at $50^{\circ} \mathrm{C}$ after 14 hours of exposure in the reference solution. At temperatures of 30 and $40^{\circ} \mathrm{C}$, three curve sections were observed. Each curve section corresponds to desorption of inhibitor particles bound by different types of metal-surfactant adsorption. The first curve section corresponds to destruction of the layer of particles formed due to weak Van der Waals interaction forces, then particles retained by electrostatic attraction are desorbed. Finally desorption of chemisorbed inhibitor that performs the protective function of the pre-adsorbed film of the surfactant occurs. Figure 7 shows that the areas of $d Z / d t$ as a function of temperature for physically adsorbed particles are much larger than similar areas for chemisorbed particles.

It should be noted that at a temperature of $30^{\circ} \mathrm{C}$ of the reference solution, the desorption of chemisorbed particles is slow. As a result, protection remains practically unchanged for nearly 45 hours. At higher temperatures, a section where the degree of protection does not depend on time is not observed.

\section{Conclusions}

1. It is experimentally shown that the curve of degree of protection $Z$ versus time $t$ for various industrial wastes PVP has three sections. The first one is probably related to desorption of inhibitor particles adsorbed by Van der Waals interactions. The second one corresponds to desorption of physically adsorbed particles, and finally, the third one, to desorption of chemisorbed particles. With increasing temperature, the lengths of the first and third sections decrease. With decreasing concentration of PVP-1 additive in the pre-adsorption solution, the second section decreases.

2. The duration of residual protective effect of PVP wastes in acid corrosion of steel linearly depends on the logarithm of inhibitor concentration in the pre-adsorption solution.

3. The near-electrode concentration of the additive desorbed from steel electrode surface is described as a function of time from the start of desorption and solution temperature. Based on this, the change in the effective activation energy of steel corrosion during inhibitor desorption was estimated as a function of time.

\section{References}

1. V. P. Grigoriev and N. A. Belousova, Vestnik TSU, 2013, 18, no. 5, 2275 (in Russian).

2. V. P. Grigoriev, N. A. Belousova and E. V. Plekhanova, Vestnik TSU, 2013, 18, no. 5, 2278 (in Russian). 
3. V. P. Grigor'ev, S. P. Shpan'ko, V. V. Boginskaya and E. V. Plekhanova, Fiz. Khim. Poverkhn. Zashch. Mater., 2010, no. 1, 88 (in Russian).

4. S. P. Shpan'ko, V. P. Grigor'ev, E. V. Plekhanova and V. A. Anisimova, Fiz. Khim. Poverkhn. Zashch. Mater., 2010, no. 2, 255 (in Russian). 